\title{
DEVELOPMENT OF EXTERNALLY POWERED UPPER EXTREMITY ORTHOTIC SYSTEMS
}

\author{
Thorkild J. Engen, ${ }^{*}$ Houston, Texas, United States of America
}

In recent years an increasing number of patients are surviving with extensive paralysis which includes the loss of hand and arm movements in both upper extremities. This disability occurs in patients with cord lesions from the fourth to the sixth cervical vertebrae who have survived with tetraplegia, as well as in patients with severe paralysis from poliomyelitis, with central nervous system degeneration, with disorders of the spinal motor system and with myopathies.

This short report describes the recent developments of a simple yet very useful externally powered orthotic system for the upper limb which resulted from a three-year research project carried out in the Department of Orthotics at the Texas Institute for Rehabilitation and Research and the Baylor University College of Medicine. The most important objective in developing this method was to restore hand and arm movements to chair-bound tetraplegic patients and others similarly handicapped with flaccid paralysis of various types.

A simplified pneumatic system controlled by the patient was developed. Not only has this approach cosmetic appeal, but it also allows the patient the dignity of a separate existence because of the direct participation of the patient in the control of the power as well as being a consciously active part of the orthotic system. Each patient has to be assessed carefully and analysed kinesiologically to determine the patterns of function that remain, the strength and usefulness of the principal muscles serving the desired integrated actions, and where the severe losses are located. The patient must be able to tolerate the sitting position at 80 to 90 degrees. Joint tightness or muscle spasm exclude a patient from this orthotic system.

Practical experience has repeatedly shown the futility of imposing upon a patient a complicated mechanism that makes him a passive robot and which does not serve a proportionately useful purpose. Such a mechanism will not be used continuously or effectively if the patient, on his own, can find any other way, even when this involves reduced function.

The three principal hand and arm actions considered to te most useful and which may be restored by this orthotic system are: 1) finger prehension, as used in grasping objects of moderate size and weight and of different shapes, such as a pencil, pen, paper or eating utensils, a glass, a cup and similar objects; 2) horizontal actions of hand and forearm, as used in writing, page turning, and similar actions not requiring very forceful actions at the shoulder joint and which can be given by slight alteration of the position of the head and trunk; and 3) smoothly combined synchronised action of the upper extremity which includes elbow flexion, shoulder flexion, vertical and horizontal abduction, and medial and lateral rotation. These movements allow patients to eat, wash their faces and do their hair.

Power actuator-The power actuator used is a McKibben muscle substitute. This consists of a straight piece of braided dacron sleeving, a gas-tight inner rubber tube with end seals for attaching to pressure tubing. When inflated it expands and shortens. Compressed carbon dioxide is used to inflate the " muscle." This power source has proved to be most practical because it is generally available and the small cylinder used by the patient can easily be refilled. Also it is non-toxic and inexpensive.

Control system-The usefulness of actions obtained with a powered orthotic system largely depends on the simplicity and efficiency of the control system, and it is desirable that the patient be in control of this system and not the system in control of the patient.

A new principle was discovered in developing a simple valve as a control device, consisting of a flexible silastic tube with a spring-loaded pinching arm capable of depressing the tube

* Director of the Orthotic Department, Texas Institute for Rehabilitation and Research, Houston, Texas, and Clinical Instructor in Orthotics, Department of Rehabilitation, Baylor University College of Medicine.

vol. 47 B, NO. 3, AUGUSt 1965 
sufficiently to stop the flow of gas under 70 pounds pressure per square inch. The valve mechanism, weighing approximately 9 grammes, is embedded in a plastic housing moulded from polyester resin. This efficient and simple valve permits the patient to do a smooth,

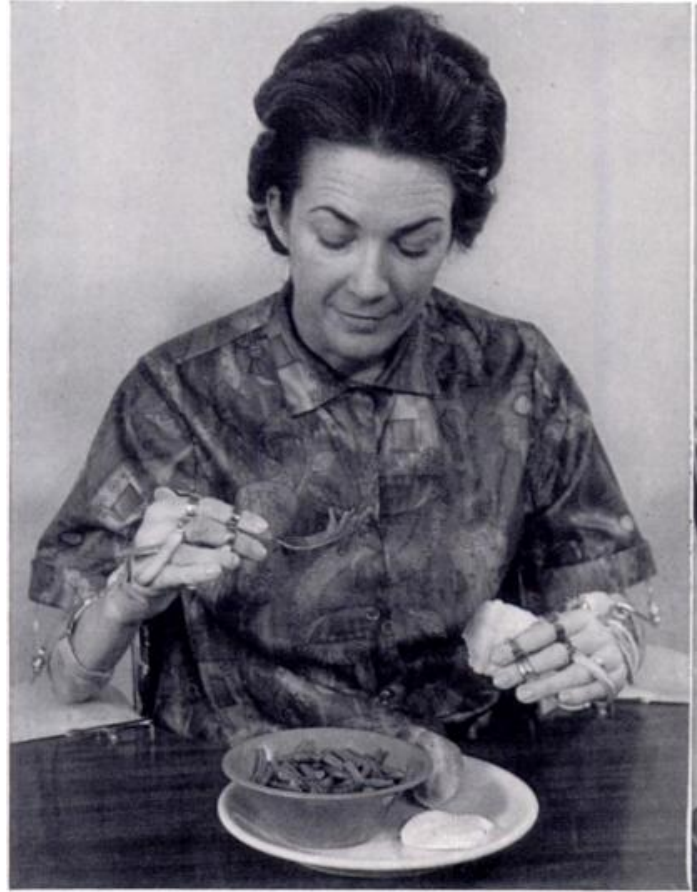

FIG. 1

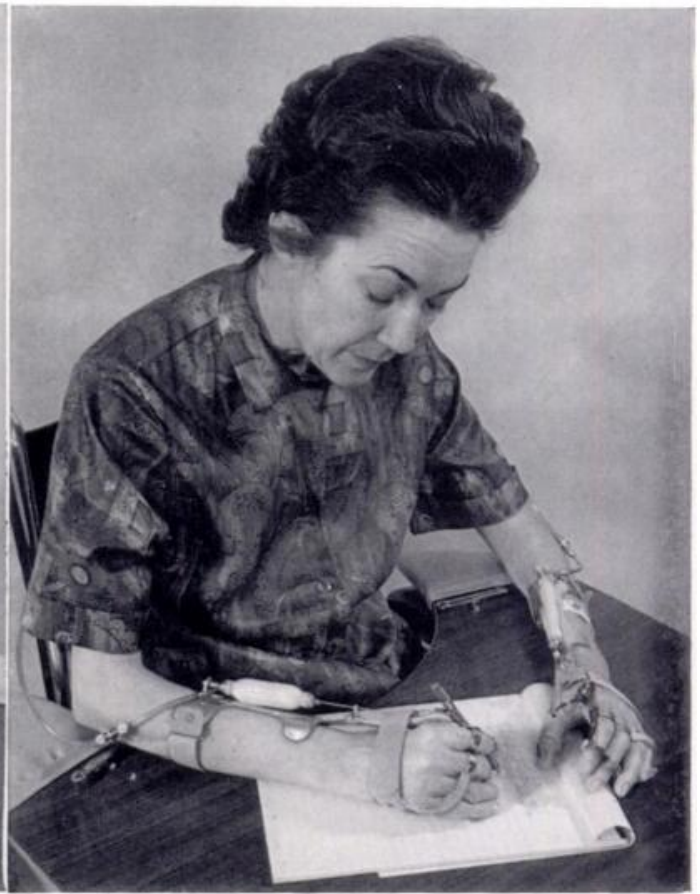

FIG. 2

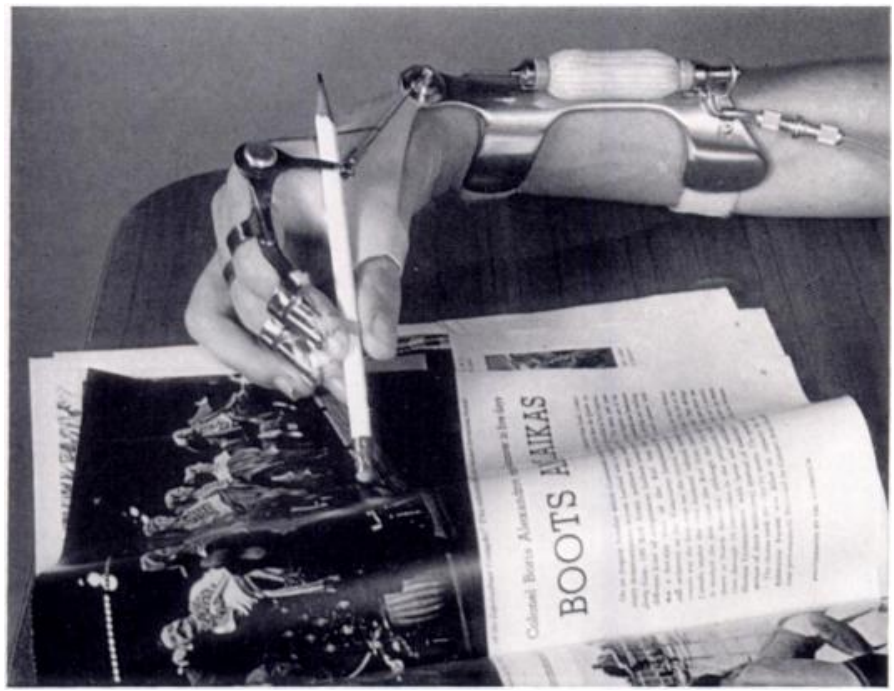

Figs. 1 To 3

Case 1. Showing various activities such as eating, writing and turning pages of a book.

FIG. 3

proportional and graduated movement. The arrangement for working the valve is selected for each patient on the availability of voluntary control movements. Any slight movement that the patient can initiate may be used to activate the system. 


\section{CLINICAL CASES}

The following illustrations which include a summary of occupational therapy assessments demonstrate two typical patients using their externally powered units.

Case 1 (Figs. 1 to 3)-A thirty-nine-year-old woman sustained a tetraplegia after transection of the spinal cord at the fifth and sixth cervical vertebrae in August 1962. She now leads a wheel-chair life in her home with the aid of bilateral external power prehensile orthoses (Figs. 1 to 3). She plans all meals, makes out grocery lists, supervises house cleaning and is able to assist her children with their studies. She takes care of her personal hygiene, applies make-up, feeds herself and handles glasses and cups with confidence. Her handwriting has shown great improvement in clarity and she now has the endurance to continue with some of the family correspondence using her external power units. Before application the patient was dependent on others for all the activities of daily living.

After application of bilateral external power units the patient is able to feed herself and can pick up a fork and spoon and position them. She is able to reach her plate, side plate,

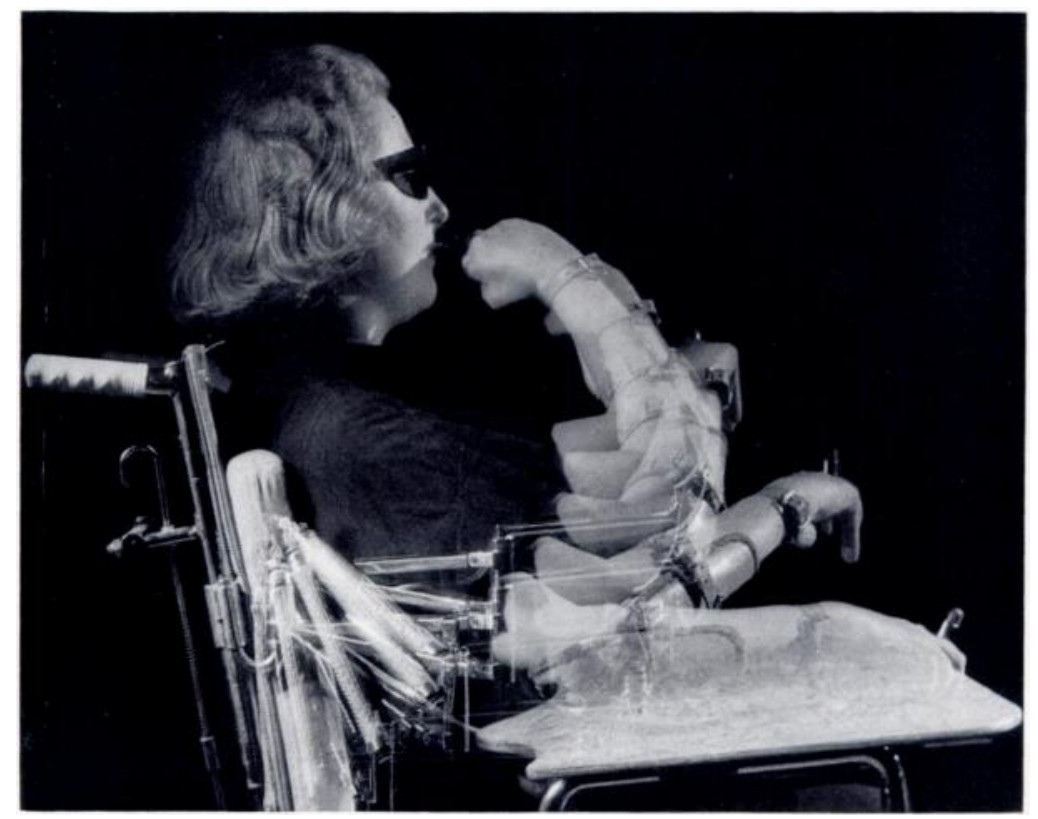

FIG. 4

Case 2-A composite photograph showing shoulder and elbow flexion.

soup bowl, etc. She can write with either pencil or pen. She uses an electric typewriter with the aid of two capped ballpoint pens and types for two hours before getting tired. She turns pages of magazines and books by using the eraser end of a pencil. She is able to telephone by holding the receiver in the left hand and dialling with a pen in the right hand. She can brush her teeth and rinse her mouth, apply make-up including powder, complexion base, eyebrow pencil, eye shadow, mascara, lipstick and the use of cleansing tissue. She can participate in games such as draughts, dominoes and cards.

Case 2-A woman of twenty-six who had poliomyelitis in September 1951 now has slight residual function of the right hand which includes radial wrist extensors, opponens of the thumb and scattered intrinsics throughout the fingers; this enabled her to grasp objects in a gross manner. Only weak wrist flexion and supination was present on the left side. Because of the complete absence of function of the elbow and shoulder on both sides it was decided,

vol. 47 B, NO. 3, AUGUST 1965 
after the initial evaluation, to apply power assistance to the right extremity to restore elbow flexion and shoulder abduction. The slight movement of the left wrist was enough to activate the two double control valves mounted on the left side of the lapboard.

Before application the patient was able to turn pages with a suspension sling applied to the right arm. This was not done smoothly or easily and needed much positioning. Smoking was possible if someone put a cigarette in a holder and flexed her arm. Typing was just possible with a right suspension sling attachment. Writing was poor, shaky and tiring. After application of the orthosis (Fig. 4) she was able to turn pages in a magazine, book and newspaper with coordination. She could smoke and put a cigarette in a holder and light it safely. She could write with a slightly built-up pencil and a raised surface for as long as three hours with brief rest periods. She was independent in feeding with an adapted fork, plate guard and lazy susan rotating disk, and was able to feed herself three times a day. She could put on make-up such as powder, rouge, mascara and lipstick, using a mirror on her lapboard and a make-up holder for the cosmetics. She could type with ease, and could insert the paper, fold the letter and insert it in the envelope. After setting up the orthosis the patient was able to work at mosaics, hook rugs, leather lacing, ceramics and to play bongo drums. She is now working as a statistical calculator.

At the present time twenty-five patients with various types of disability are using one, or a combination, of the described orthotic systems, and are being evaluated at intervals. In addition to being able to undertake activities of daily living, most of these patients are engaged in some form of vocation.

\section{SUMMARY}

1. Orthotic systems for the upper limb are described.

2. Two patients are described to illustrate the value of the systems.

From 1960 to the present most of this work has been supported by a Research Grant from the Vocational Rehabilitation Administration No. RD542 to Baylor University College of Medicine. 